\title{
Curvature-dependent tension and tangential flows at the interface of motility-induced phases
}

\author{
Adam Patch, ${ }^{* a}$ Daniel Sussman, ${ }^{a}$ David Yllanes, ${ }^{a, b, c}$ and M. Cristina Marchetti ${ }^{a, c}$
}

Received Date

Accepted Date

DOI: $10.1039 / x x x x x x x x x x$

www.rsc.org/journalname

\begin{abstract}
Purely repulsive active particles spontaneously undergo motility-induced phase separation (MIPS) into condensed and dilute phases. Remarkably, the mechanical tension measured along the interface between these phases is negative. In equilibrium this would imply an unstable interface that wants to expand, but these out-of-equilibrium systems display long-time stability and have intrinsically stiff boundaries. Here, we study this phenomenon in detail using active Brownian particle simulations and a novel frame of reference. By shifting from the global (or laboratory) frame to a local frame that follows the dynamics of the phase boundary, we observe correlations between the local curvature of the interface and the measured value of the tension. Importantly, our analysis reveals the presence of sustained local tangential motion of particles within a surface layer in both the gas and the dense regions. The combined tangential current in the gas and self-shearing of the surface of the dense phase suggest a stiffening interface that redirects particles along itself to heal local fluctuations. These currents restore the otherwise wildly fluctuating interface through an out-of-equilibrium Marangoni effect. We discuss the implications of our observations on phenomenological models of interfacial dynamics.
\end{abstract}

\section{Introduction}

Pierre-Gilles de Gennes wrote $\mathrm{e}^{1}$ that "the interfaces between two forms of bulk matter are responsible for some of the most unexpected actions... the overlap region is mobile, diffuse, and active." This description is particularly apt as applied to the emergent behavior of dense collections of active Brownian particles (ABPs), in which purely repulsive particles are driven out of equilibrium via self-propulsive forces in an overdamped environment ${ }^{2}$. Even in the absence of attractive interactions, such systems can spectacularly phase separate into a dense liquid phase coexisting with a dilute gaseous phase $\mathrm{e}^{2}$. This motility-induced phase separation (MIPS) is heuristically understood by considering the persistent dynamics of an individual particle, and it occurs when the time for a particle to re-orient after a collision becomes long relative to the typical mean free time between those collisions. The occurrence of MIPS has also been described through an approximate mapping onto an effective equilibrium system undergoing conventional vapor-liquid phase separation. ${ }^{[-13}$ It has been demon-

\footnotetext{
${ }^{a}$ Department of Physics and Soft and Living Matter Program, Syracuse University, Physics Building, Syracuse, New York 13244, USA

${ }^{b}$ Instituto de Biocomputación y Física de Sistemas Complejos (BIFI), 50009 Zaragoza, Spain

${ }^{c}$ Kavli Institute for Theoretical Physics, University of California, Santa Barbara, CA 93106, USA

*apatch@syr.edu
}

strated in numerical simulations in two and three dimensions for various minimal models and repulsive potentials. 2[14]17] Experiments in active colloids and bacterial suspension, however, generally observe the formation of finite-size clusters rather than bulk phases, suggesting that non-generic phenomena may be at play and arrest the phase separation. 18.23

A typical snapshot of such an out-of-equilibrium phase separation is shown in Fig. 1, where the enormous fluctuations characteristic of MIPS are readily seen. Large fluctuations occur both at the interface and in the bulk of the dense phase, where bubbles of the dilute phase spontaneously nucleate and travel to the phase boundary, breaking at the surface (see SI for a video). Despite the wildly fluctuating nature of the interfaces, connections with interfacial properties of equilibrium phases have been identified. $12[13|24| 28$ For example, as we describe in more detail below, the scaling of the interfacial stiffness with system size is found to be consistent with equilibrium arguments. There is, however, a major caveat: the measured interfacial tension $\gamma$ is negative, $13.24 / 26$ and the equilibrium arguments connecting it to an interfacial the stiffness require one to take $|\gamma|$ as the relevant quantity. $\frac{25}{25}$

How can we reconcile a stable, equilibrium-like interface with negative values of surface tension, especially in a system driven by far-from-equilibrium dynamics? In this work we rely on extensive simulations to study the structure of the MIPS interface. 


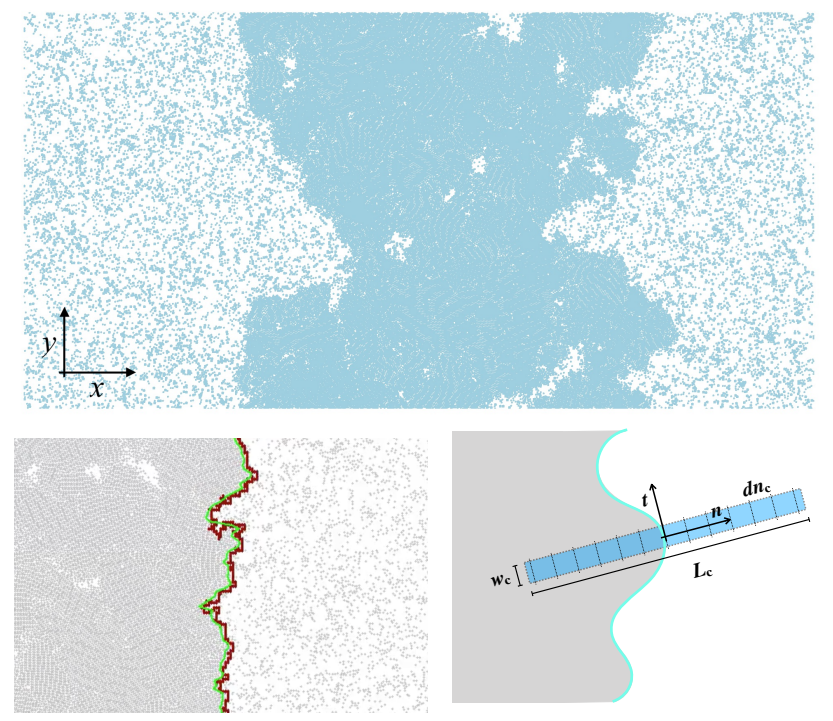

Fig. 1 Top: A snapshot of a system composed of roughly $2 \times 10^{5}$ active Brownian particles of radius $r_{0}$ undergoing spontaneous separation into dense and dilute phases. The persistence length of the particles (defined in the text) is $\ell_{p}=100 r_{0}$; the area fraction is $\phi=0.5$ and phase separation into a strip geometry is attained by choosing the aspect ratio of the simulation box to be $L_{x} / L_{y}=2$, with periodic boundary conditions (here $L_{x}=1600 r_{0}$ ). Bottom left: A demonstration of two methods for identifying the interface. The red curve results from a contour-finding algorithm that captures all overhangs and allows for local curvature measurements, while the green curve considers only the outermost particles at each value of $y$ and can be used to obtain the spectrum of the interface height fluctuations (see Appendix $\mathrm{A}$ for details). Bottom right: $\mathrm{A}$ schematic of the the local frame we use to measure dynamical quantities near the interface (see Appendix B for details).

We show that for such strongly fluctuating interfaces - where the instantaneous deviation of the interfacial height from its average value is decidedly not small - a transformation to the local coordinate frame along the interface (illustrated in Fig. 1) reveals surprising additional structure. An important finding is a strong correlation between the local curvature and the magnitude of the surface tension. By examining in detail the local dynamics near the fluctuating interface, we demonstrates the existence of surface layers with large local tangential particle motion in both the dilute and the dense regions. While such tangential currents in the interfacial region within the gas have been highlighted before, the self-shearing of the surface layer in the dense phase is a new result. This observation suggests a new mechanism for the stabilization of active interfaces where the interface directs particles along itself to heal fluctuations.

Below, we first describe our simulation model and study the properties of the interface in a global reference frame, in line with other studies. 13[24 26 In addition to expanding on previous results for the scaling of the interfacial width with simulation size, we directly study the spectral density of the interface fluctuations. Despite the non-equilibrium character of the system, this spectrum is surprisingly equilibrium-like. In this global frame we also study the (mechanically defined) value of the interfacial tension over a wide range of parameters, varying the persistence length of the particles. We confirm the unusual result that the interfacial width scales with a stiffness proportional to the absolute value of a negative $\gamma$.

We then shed new light on the mechanism of interfacial stability by shifting to a local frame defined along the interface, utilizing an algorithmically traced interface contour with single particle resolution. In this frame, it is straightforward to define normal and transverse particle fluxes and forces. Using this technique we quantify the strong correlations between the local curvature of the interface and the value of the surface tension: although we find that the tension is always negative, it is closest to zero in regions of large positive curvature (inward "valleys") and most strongly negative in regions of large negative curvature (outward "peaks").

We close by presenting a heuristic picture of the emergent collective behavior of self-propelled particles near the interface, highlighting how their dynamics produces a negative interfacial "tension", resulting in an effectively "extensile" interface that tends to grow longer, while maintaining its integrity. This is in sharp contrast with familiar equilibrium interfaces that are "contractile" in the sense that the positive tension always tends to shorten the interface. These interfaces have been phenomenologically interpreted in terms of Edwards-Wilkinson-like growth processes, 2629 but the dependence of the surface tension on local curvature naturally leads to simple KPZ-like equations. ${ }^{[30}$ We attempt different scaling collapses of our data for interfacial roughening in an effort to discriminate between these scenarios.

\section{Model and Methods}

We simulate a system of active Brownian particles (ABPs) in a regime in which they are known to undergo motility-induced phase separation. We choose a strip geometry for ease of identification of the interface. We identify the dense phase as all members of the largest set of touching particles, while the gas phase is composed of those remaining particles.

\subsection{Active Brownian Particle Model}

A minimal model of monodisperse, purely repulsive $\mathrm{ABPs}^{2}$ consists of $N$ self-propelled particles with interaction radius $r_{0}$. We place these in a rectangular simulation domain of sides $L_{x}$ and $L_{y}$ with periodic boundary conditions, setting $L_{x} / L_{y}=2$ so that the bulk phases yield a quasi-1D interface (see Fig. 1). Each particle is identified by its position $\mathbf{r}_{i}$ and director $\hat{\mathbf{e}}_{i}=\left(\cos \theta_{i}, \sin \theta_{i}\right)$ that defines the direction of the propulsive force $\mathbf{F}_{i}^{\mathbf{S}}=\left(v_{0} / \mu\right) \hat{\mathbf{e}}_{i}$, where $v_{0}$ is the propulsion speed and $\mu$ the mobility (inverse friction). The particles are governed by the overdamped Langevin equations

$$
\begin{aligned}
& \dot{\mathbf{r}}_{i}=\mu\left(\mathbf{F}_{i}^{\mathrm{s}}+\sum_{j \neq i} \mathbf{F}_{i j}\right), \\
& \dot{\theta}_{i}=\sqrt{\frac{2}{\tau_{\mathrm{r}}}} \eta_{i}(t),
\end{aligned}
$$

where $\eta_{i}(t)$ is a Gaussian random torque with zero mean and variance $\left\langle\eta_{i}(t) \eta_{j}\left(t^{\prime}\right)\right\rangle=\delta_{i j} \delta\left(t-t^{\prime}\right)$. The variance of this fluctuating torque is set by the persistence time $\tau_{\mathrm{r}}=1 / D_{\mathrm{r}}$, where $D_{\mathrm{r}}$ is rotational diffusion. The pair forces between particles $i$ and $j$, $\mathbf{F}_{i j}$, are obtained from a repulsive Weeks-Chandler-Anderson po- 
tential, $\mathbf{F}_{i j}=-\frac{\partial V_{\mathrm{WCA}}}{\partial r_{i j}}=\frac{12 \varepsilon}{r_{\mathrm{c}}}\left[\left(\frac{r_{\mathrm{c}}}{r_{i j}}\right)^{13}-\left(\frac{r_{\mathrm{c}}}{r_{i j}}\right)^{7}\right] \hat{\mathbf{r}}_{i j}$ for $r<r_{\mathrm{c}}=2 r_{0}$ and $\left|\mathbf{F}_{i j}\right|=0$ otherwise, where $\mathbf{r}_{i j}=\mathbf{r}_{i}-\mathbf{r}_{j}$ is the interparticle separation and $\hat{\mathbf{r}}_{i j}=\mathbf{r}_{i j} /\left|\mathbf{r}_{i j}\right|$.

We neglect noise in the translational dynamics, which is less important than the orientational noise in both synthetic active colloids and swimming bacteria. $\frac{23 \mid 31}{2}$ The non-equilibrium nature of this active model is provided entirely by the propulsive force $\mathbf{F}_{i}^{\mathrm{s}}$ of each particle. After integrating out the angular dynamics, $\mathbf{F}_{i}^{\mathbf{s}}$ represents a non-Markovian stochastic force correlated over the persistence time $\tau_{\mathrm{r}}$. Since the finite correlation time of the noisy propulsive force is not matched by similar correlations in the (constant) mobility $\mu$, the system does not obey the fluctuationdissipation theorem embodied by the Stokes-Einstein relation.

The persistence time $\tau_{\mathrm{r}}$ controls the crossover from ballistic to diffusive single particle dynamics: at short times the dynamics of non-interacting ABPs is ballistic, and for $t \gg \tau_{r}$ it is diffusive with diffusion coefficient $D_{\mathrm{s}}=v_{0}^{2} \tau_{r} / 2$. The single-particle dynamics can be characterized by the persistence length $\ell_{\mathrm{p}}=v_{0} \tau_{\mathrm{r}}$, which, together with the area fraction $\phi$, controls the phase behavior of this interacting non-equilibrium system. 431

\subsection{Capillary waves and interface width}

Taking a mesoscopic view of the interface, one can characterize the fluctuations in terms of the deviations of the instantaneous location of the surface along the $x$ direction from its mean value,, 32 $\delta h(y, t)=h(y, t)-\bar{h}(t)$, with $\bar{h}(t)=\frac{1}{L_{y}} \int_{0}^{L_{y}} d y h(y, t)$. The mean interfacial width can then be written as,

$$
w^{2}=\frac{1}{L_{y}} \int_{0}^{L_{y}} \mathrm{~d} y\left\langle|\delta h(y)|^{2}\right\rangle=\sum_{q}\left\langle|\delta h(q)|^{2}\right\rangle,
$$

where

$$
\delta h(q)=\frac{1}{L_{y}} \int_{0}^{L_{y}} \mathrm{~d} y \delta h(y) \mathrm{e}^{-\mathrm{i} q y} .
$$

In thermal equilibrium, interfaces carry an excess free energy $E_{\mathrm{s}}=\gamma \ell$ determined by the constant interfacial tension, $\gamma$, and the length $\ell$ of the interface, with

$$
\ell=\int_{0}^{L_{y}} \mathrm{~d} y \sqrt{1+\left|\nabla_{y} h(y)\right|^{2}} \approx L_{y}\left[1+\frac{1}{2} \sum_{q} q^{2}|\delta h(q)|^{2}\right] .
$$

The interfacial height mode amplitudes are then determined by the equipartition theorem as

$$
\left\langle|\delta h(q)|^{2}\right\rangle=\frac{2}{L_{y}} \frac{k T}{\gamma q^{2}} .
$$

Eq. (3), we can immediately calculate the interface width as

$$
w^{2}=w_{0}^{2}+\sum_{q>0}\left\langle|\delta h(q)|^{2}\right\rangle=w_{0}^{2}+\frac{L_{y}}{12 \sigma},
$$

where $w_{0}^{2}$ describes the fluctuations of the $q=0$ mode and $\sigma=$ $\gamma / k T$ is the interfacial stiffness that measures the cost of deformations along the entire length $L_{y}$.

\subsection{Interfacial tension}

A mechanical definition of the interfacial tension $\gamma$ can be obtained by examining the work $\delta W=\gamma \delta \ell$ needed to change the length of the interface by an amount $\delta \ell$. Here we follow the standard quasi-thermodynamic treatment of Ref. 32 , where in equilibrium it is shown that this mechanical definition yields the same value as that obtained from the interfacial fluctuations. As we will see, this is not, however, the case for ABPs $24 \mid 26$.

Working in two dimensions, we consider a one-component system confined to a box of area $A=\ell^{2}$ and separated into two bulk phases, with a vertical interface at some position $0<x_{0}<\ell$. If the area of the system is changed isotropically by an amount $\delta A$, the associated work is controlled by the pressure, with $\delta W=-p \delta A$. To define the tension we imagine isothermally and reversibly deforming the sides of the confining box so that the interface increases in length by an amount $\delta \ell$, while maintaining fixed area. This requires an anisotropic deformation of the box, but the symmetry of the interface ensures that the pressure tensor can only have non-zero components $p_{x x}$ and $p_{y y}$. The tangential work done to the system in increasing the length of the interface is then

$$
\delta W_{t}=-\delta \ell \int_{0}^{\ell} \mathrm{d} x p_{y y}(x)
$$

and the normal work done in keeping the area fixed is

$$
\delta W_{n}=\ell \delta \ell p_{x x}
$$

In writing the above expression we have assumed mechanical stability of the interface, $\nabla \cdot \mathbf{p}=0$. This, together with the symmetry of the system ensures that $p_{x x}$ is not itself a function of $x$ or $y$. The total work done is then

$$
\delta W=\delta W_{n}+\delta W_{t}=\delta \ell \int_{0}^{\ell} \mathrm{d} x\left(p_{x x}-p_{y y}(x)\right) .
$$

Comparing this expression with $\delta W=\gamma \delta \ell$ we recover the Kirkwook-Buff expression 33 ,

$$
\gamma=\int_{\text {dense }}^{\text {dilute }} \mathrm{d} x\left[p_{\mathrm{n}}-p_{\mathrm{t}}(x)\right]
$$

where we have more generally replaced Cartesian components of the tensor with normal and tangential components, and assumed that the two phases we are considering are a dense and dilute phase.

Equation 11 quantifies the surface tension as the total anisotropy in pressure across the interface. It assumes of course that the local pressure tensor has a mechanical definition. In the next section we outline how we measure the local pressure tensor for our active system and compute Eq. 11

\subsection{Pressure in Active Matter}

The pressure $p$ of a system of overdamped torque-free active particles involves two contributions: a contribution due to direct particle interactions, $p_{\mathrm{d}}$, and a "swim" contribution that represents the flux of propulsive forces across a unit area, $p_{\mathrm{s}}$, with $p=p_{\mathrm{d}}+p_{\mathrm{s}}$. The components of the interaction pressure tensor $p_{\mathrm{d}}^{\alpha \beta}$, where $\alpha, \beta$ denote Cartesian indices, are given by a virial 

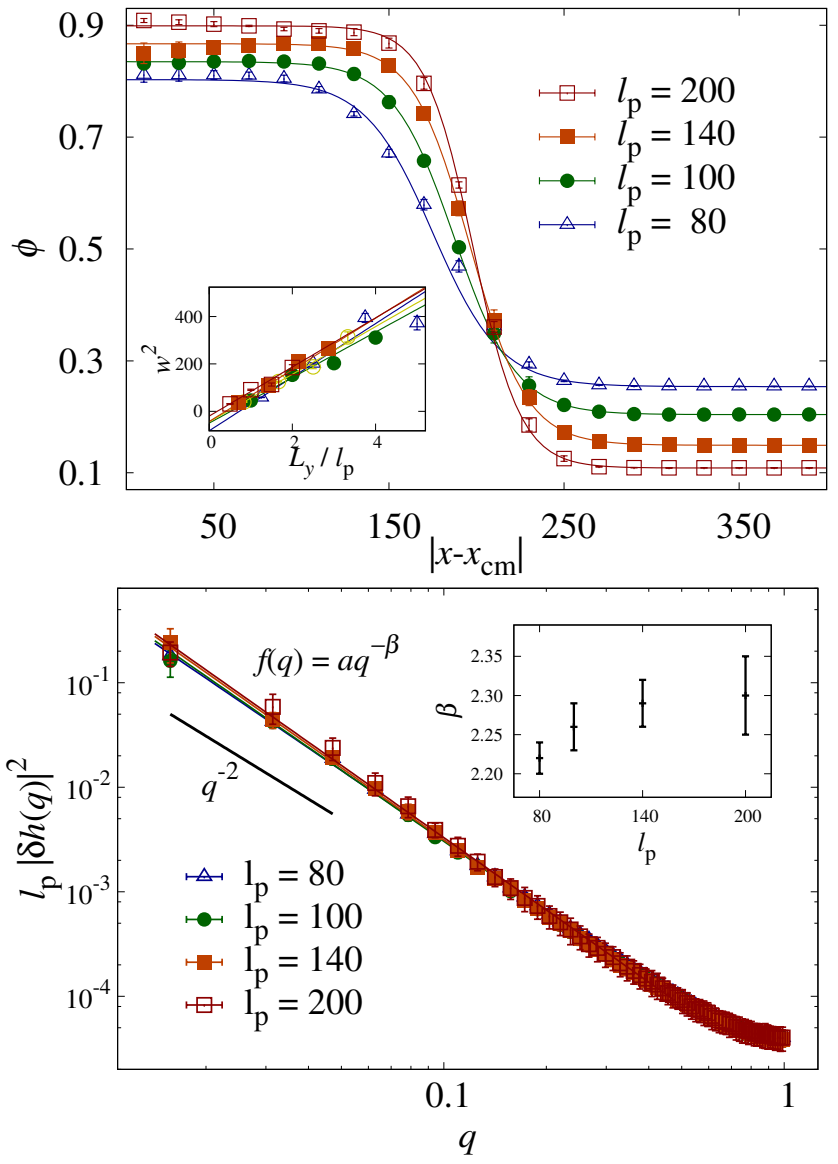

Fig. 2 Top: density profiles in the interface frame, for varying persistence. [Inset] Scaling of $w^{2}$ as a function of $L_{y} / \ell_{\mathrm{p}}$, for comparison to Eq. 7 Bottom: Interface height fluctuation spectrum for systems with $L_{y}=400$, collapse for several values of $\ell_{\mathrm{p}}$. The equilibrium expectation $\left\langle|\delta h(q)|^{2}\right\rangle \sim q^{-2}$ is shown as a solid black line. The collapse of $\ell_{\mathrm{p}}|\delta h(q)|^{2}$, signifies the clear dependence of interfacial fluctuations on persistence. [Inset] Best fit exponent characterizing the initial decay of the interface modes as a function of $\ell_{\mathrm{p}}$.

expression in terms of particle pair interactions,

$$
p_{\mathrm{d}}^{\alpha \beta}=\frac{1}{d A} \sum_{i, j} F_{i j}^{\alpha} r_{i j}^{\beta} .
$$

The swim pressure can also be calculated via a virial-like expression. 163134 A numerically more useful expression for the local the swim pressure is given in terms of the flux of "active impulse", as

$$
p_{\mathrm{s}}^{\alpha \beta}=\frac{1}{d A} \sum_{i} J_{i}^{\alpha} v_{i}^{\beta},
$$

where $\mathbf{v}_{i}$ is the velocity of particle $i$ and $\mathbf{J}_{i}\left[\theta_{i}(t)\right]=\mathbf{F}_{i}^{s}(t) \tau_{\mathrm{r}}$ is the

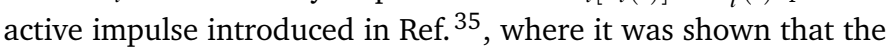
expression given in Eq. (13) for the swim pressure is equivalent to the virial one proposed in previous work. $\frac{16 \mid 36}{1}$ The form in term of active impulse is more convenient for numerical studies because it avoids the strong finite size effects that arise in calculations of the virial expression for the swim pressure. $\frac{34}{3}$

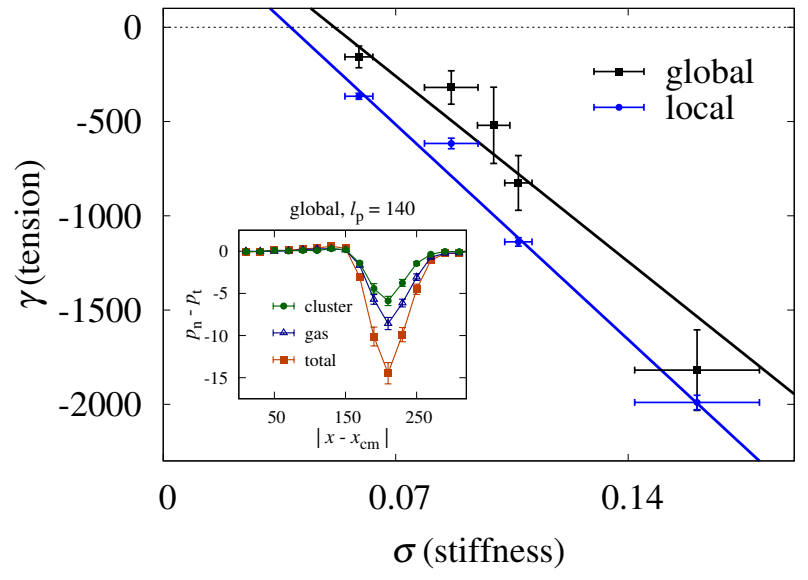

Fig. 3 The main figure correlates the interfacial tension and the stiffness, showing that the tension becomes more negative with increasing stiffness. This suggests that the same dynamics that produces a positive stiffness yields a negative interfacial tension. The black points correspond to measurements in the global frame (described in Sec. 3, while the blue points correspond to measurements in the local frame of the interface (described in Sec. 4). The inset shows the difference between normal and tangential pressure that determines the integrand in Eq. 11 broken down into cluster and gas contributions, highlighting the significant net-negative contributions from both cluster and gas particles.

\subsection{Simulations}

Simulations of large phase-separating systems of ABPs require total running times $t \gg \tau_{\mathrm{r}}$ and integration timesteps $\Delta t \ll \tau_{\mathrm{r}}$. ${ }^{[34}$ This, in addition to the wildly fluctuating nature of the interfaces and the need to sweep different $\ell_{\mathrm{p}}$ values, means that considerable computational effort must be made to gather an adequate statistical sample. Most of our simulations have been carried out on GPUs using the HOOMD-blue $\frac{37 / 38}{3 i m u l a t i o n ~ p a c k a g e, ~ c o m p l e-~}$ menting with CPUs for the smaller system sizes.

To suppress transients, we nucleate the phase-separated strip by initially placing particles in a triangular lattice with interparticle distance $r_{i j}=2 r_{0}$. We performed parameters sweep by using persistence lengths $\ell_{p}=\{60,80,100,120,140,200\}$ and system sizes $L_{x}=\{200,400,600,800,1600\}$. The area fraction was fixed at $\phi=0.5$, with $\mu=1$ and $v_{0}=100$. We have simulated for $t \geq 1 \times 10^{3} \tau_{\mathrm{r}}$, with a number of independent runs $N_{\text {runs }}=100$ for our smallest systems, down to $N_{\text {runs }}=8$ for $L_{x}=800$. Unless stated otherwise, the results in this paper refer to $L_{x}=800$ systems. In order to study the interface growth, we have also run shorter simulations $\left(t=100 \tau_{\mathrm{r}}\right)$ that do not reach the stead state, for system sizes ranging from $L_{x}=400\left(N_{\text {runs }}=400\right)$ to $L_{x}=2400$ $\left(N_{\text {runs }}=28\right)$.

We note that previous studies have found strong finite-size effects when studying the pressure of phase-separated systems with $\ell_{\mathrm{p}} \sim L$, even at low density. $\frac{34}{3}$. Measuring the swim pressure with the active impulse flux, Eq. (13), rather than with a virial expression, mitigates this effect. Nevertheless, we have kept the values of $\ell_{p}$ smaller than $L_{x}$ in our work.

\section{Measurements in the global frame}

The MIPS interface is characterized by wild fluctuations (as seen in Fig. 1). Upon time-averaging, however, the interface appears 
deceptively well behaved and equilibrium-like. In this section we show this by characterizing the average density profile, determining the stiffness of the interface from the scaling of the interfacial width, and investigating the spectrum of interface fluctuations. We will often refer to the system as divided into two sets of particles: those belonging to the largest connected cluster (dense) and those in the gas (dilute).

\subsection{Interface width and stiffness}

Working in the global frame, we first consider the average area fraction projected onto the $x$-axis, $\phi(x)=\frac{1}{L_{y}} \int_{0}^{L_{y}} \mathrm{~d} y \phi(x, y)$. The resulting profile is well described by

$$
\phi(x)=\frac{\phi_{+}+\phi_{-}}{2}+\frac{\phi_{+}-\phi_{-}}{2} \tanh \left(\frac{x-x_{0}}{2 w}\right),
$$

where $\phi_{ \pm}$denotes the area fraction for the dense and dilute phases, $x_{0}$ is the mean position of the interface, and $w$ is a measure of the interfacial width. In broad agreement with previous observations, ${ }^{5}$ we find that with increasing $\ell_{\mathrm{p}}$ the interfacial width decreases and the difference in density between the coexisting phases increases, as we show in the upper panel of Fig. 2. The inset to the upper panel of Fig. 2 also shows that $w^{2} \sim L_{y}$ for a variety of $\ell_{\mathrm{p}}$.

We note that the finite intercept at $L_{y}=0$ provides an estimate of the minimum system size needed to observe MIPS in these systems. As shown in Section 2, Eq7 connects the scaling of the interfacial width with system size $L_{y}$ to the intrinsic stiffness of the interface, $\sigma$ that can be extracted from the slope of the linear curves in the inset.

\subsection{Interface fluctuations}

Although the MIPS interface is extremely rough and is characterized by frequent overhangs, to quantify the structure of the interfacial fluctuations we approximate the interface by constructing a height map $h(y)$ as described in Appendix A We then examine the Fourier spectrum of $\delta h(y)=h(y)-\bar{h}$, where $\bar{h}$ is the instantaneous value of the mean position of the height map, and average the resulting mode spectrum $\left\langle|\delta h(q)|^{2}\right\rangle$ over time in the steady state. The spectrum of interfacial fluctuations shown in Fig. 2 is well described by $\left\langle|\delta h(q)|^{2}\right\rangle \sim \ell_{\mathrm{p}}^{-1} q^{-\beta}$, in keeping with our finding that the interfacial width itself scales with $\ell_{\mathrm{p}}$. We do observe small deviations from the strictly equilibrium expected scaling of $\beta=2$ (see inset of lower panel of Fig. 2).

Interfacial fluctuations have recently been measured in a mixture of active and passive disks with attractive interactions. $\frac{39}{3 n}$ In that case the attractions stabilizes the interface and activity enhances the stiffness that grows linearly with $\ell_{\mathrm{p}}$.

\subsection{Mechanical surface tension}

As discussed in Section 2, a straightforward mechanical measurement of the surface tension involves integrating differences in the local pressure tensor across the interface, 24 via $\gamma=\int \mathrm{d} x\left(p_{\mathrm{n}}-p_{\mathrm{t}}\right)$ (Eq. 11). In the global frame one simply has $p_{\mathrm{n}}=p_{x x}$ and $p_{\mathrm{t}}=p_{y y}$. At an equilibrium liquid-gas interface the positive surface tension arises from the lowering of tangential pressure associated with the weaker binding of liquid surface molecules as compared to bulk liquid molecules. In our active system, in contrast, we find a large increase of tangential pressure at the interface, as shown in the inset of Fig. 3 (see also Fig. 9 for further details). This large tangential pressure is responsible for the negative value of the interfacial tension. It arises not only from the swim pressure of the gas (Fig. 9), as shown in previous work, 132426 but also from continuous tangential self-shearing motions of particles at the surface of the dense phase. In other words, particles moving tangentially in the interfacial region both inside and outside the dense phase contribute the the negative sign of the tension, as explicitly demonstrated below.

Interestingly, we also find that the magnitude of the surface tension increases with persistence length, meaning that in these systems stiffer, sharper interfaces correspond to more negative values of tension. Classically, a negative interfacial tension would indicate an interface that prefers to grow; here the opposite occurs. Refs. $24\left[25\right.$ conjectured a "housekeeping work" $w_{\text {hk }}$ that accounts for the work done by the particle as opposed to the work done to the particle $w_{\mathrm{ex}}$, resulting in a relationship between tension and stiffness of the form $\gamma=-\sigma F^{\mathrm{s}} \ell_{\mathrm{p}}$. Earlier we found that $\sigma$ is linear in $\ell_{\mathrm{p}}$, implying a quadratic dependence of $\gamma$ on $\ell_{\mathrm{p}}$ (or on $\sigma$ ). However, the fact that $\sigma\left(\ell_{\mathrm{p}}\right)$ has a non-zero axis intercept (Fig. 2) makes it very difficult to verify this relation quantitatively. Indeed, while our results show qualitative agreement with the idea that $F^{\mathrm{s}} \ell_{\mathrm{p}}$ sets an energy scale relevant to determining interfacial stability, with our error bars $\gamma$ seems to be adequately represented by a linear dependence on $\sigma$ (Fig. 3).

\section{Measurements in the local frame}

It is well known that even in an ideal gas of ABPs the geometry of any confining wall has a strong influence on both the structure and dynamics of the system. 4041 In MIPS the gas particles interact with an emergent, self-generated boundary that continuously absorbs and releases particles, with zero net flux at steady state, but substantial local tangential currents. It is therefore illuminating to probe the interfacial structure and dynamics with respect to a local frame at each point of the interface. As described in more detail in Appendix A. we use a contour-finding algorithm to move beyond the height-map representation $h(y)$ to the position of the interface with respect to the contour length along the interface itself, $h(s)$. To do so, we define a local frame whose origin is set at points along the contour and whose orientation is set by the local normal $\mathbf{n}$ (which is also calculated using this contour by fitting a region around a given point to a quadratic function and evaluating its curvature). We then compute the average value of the pressure tensor in slices of fixed width at each point along the interface. Although our results are quantitatively sensitive to the choice of slice width and curvature coarse-graining scale, we have confirmed that our qualitative results are robust to any sensible choice of these parameters.

\subsection{Dynamics along the interface}

We first illustrate our findings by looking at the structure and dynamics of the particles in this local frame of measurement. The 


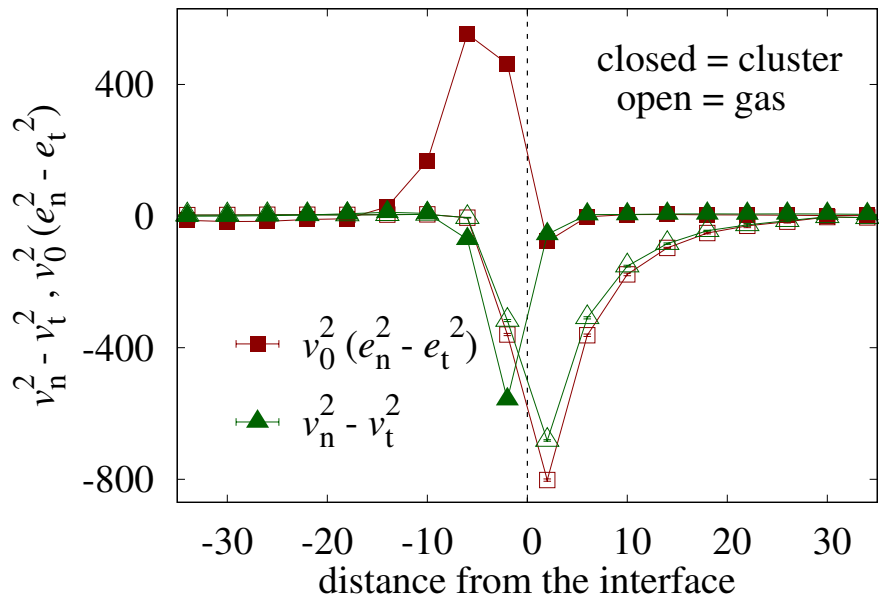

Fig. 4 We use the local frame along the phase boundary to illuminate the complex dynamics near the interface-in particular the anisotropy of director and velocity. The $x$-axis shows the distance from the interface measured along the normal, as shown in Fig. 1 Near the interface, the average director field shows an excess of inward pointing swim force just inside the cluster and an excess of transverse swim force just inside the gas. The anisotropy of the resulting motion shows that the gas moves with its director field, but the cluster self-shears as its surface particles move tangentially.

stability of MIPS interfaces has often been heuristically explained by particles pointing "inward" at the outer edge of the dense cluster, with a rotational re-orientation time for these particles that is longer than the typical time for a gas particle to arrive at the interface. ${ }^{[5]}$ The condition of net zero flux then suggests large transverse currents in the gas phase outside the dense cluster. $\frac{39}{39}$

In Fig. 4 we can see this dynamics at play in the local frame of the interface. The square points show the average anisotropy of the director, $e_{\mathrm{n}}^{2}-e_{\mathrm{t}}^{2}$, and show that indeed particles in the dense cluster but near the interface preferentially point inwards, while particles in the gas phase preferentially point tangent to the local interface. An examination of the velocity field itself, $v_{\mathrm{n}}^{2}-v_{\mathrm{t}}^{2}$, reveals, however, an unexpected behavior. In the gas phase the velocity field and the director point in the same direction as interactions are negligible, but they are distinctly different for particles at the interface within the dense phase. One clearly sees that there is a local transverse current even in the dense phase, as the projection of the swim force in the tangential direction causes the clustered particles to slide along the very boundary they are defining.

The transverse swim current is also enhanced by persistence, which we have shown corresponds to stiffer interfaces. Additional details are given in Appendix $\mathrm{C}$ where we examine the behavior in the interfacial layers both within the dense and the gas phases. One observes tangential particle motions in both regions and an associated local stiffening of the interface, with the fluctuating boundary providing a local guiding effect on active gas particles similar to that observed for curves solid walls. 41 .

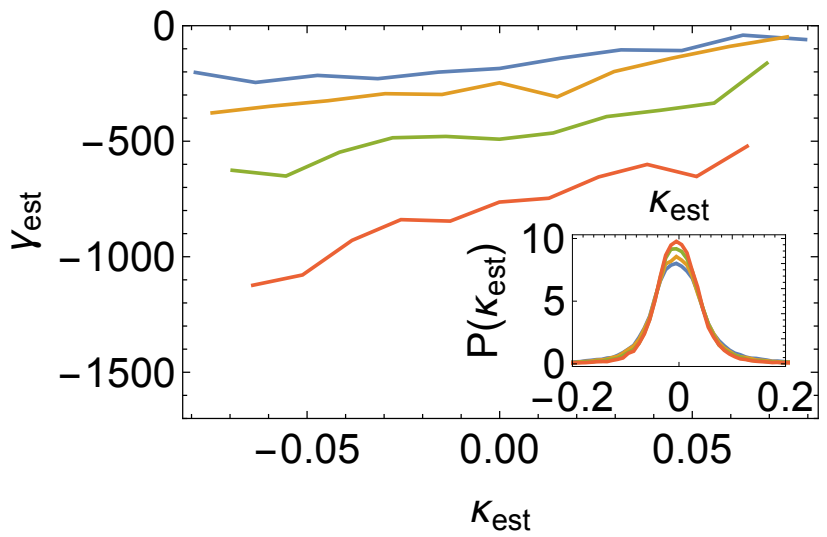

Fig. 5 Correlation between the estimated local surface tension and local curvature for $\ell_{p}=80,100,140,200$ (top to bottom). Negative and positive curvature values correspond to mountains and valleys of the interface, respectively. The correlation between curvature and tension provides a Marangoni-like effect which allows the interface to stabilize itself. [Inset] Probability distribution of local curvatures for the same parameters (where higher values of $\ell_{\mathrm{p}}$ have a more peaked distribution of curvature).

\subsection{Interfacial curvature and local surface tension}

The findings above, showing correlated flows in both the dense and dilute phases on either side of the interface, qualitatively suggest a Marangoni-like local mass transport that stabilizes the interface. Inspired by this, we explicitly investigate the connection between the curvature of $h(s)$ and the local mechanical measurement of surface tension.

In the local frame, we still find on average a negative value for the surface tension. In this frame, though, we find that the spatial profiles of $p_{\mathrm{n}}-p_{\mathrm{t}}$ are much sharper, and individual slices can be binned according to the local value of curvature. These measurements have enormous fluctuations (with a variance at least an order of magnitude larger than the mean), but careful averaging allows us to distinguish a clear correlation between local curvature and local tension, which we show in Fig. 5 (restricting ourselves to values of the local curvature within one standard deviation of the mean to ensure sufficient statistics). Although the mean surface tension is negative for all values of curvature, we find that the outward bulges of the interface (i.e., regions of negative curvature) are quantitatively more unstable than the inward valleys. This gradient of stability provides a mechanism for the interface to dynamically stabilize itself as transverse currents from the bulges on average fill in the gaps in the valleys.

\section{Discussion}

In a model of purely repulsive active Brownian particles undergoing motility induced phase separation, we have explored the surprising dichotomy of an interface that on one hand exhibits some equilibrium-like properties (a well-behaved time-averaged density profile and a nearly $q^{-2}$ spectrum of interfacial fluctuations), but on the other is governed by strong fluctuations driven by nonequilibrium physics, resulting in negative interfacial tension. It is tempting to try to write down a phenomenological model of the interface. Some authors have in fact proposed mapping the MIPS 
interface to an Edwards-Wilkinson (EW) growth model. ${ }^{26}$ On the other hand, our finding that the surface tension is itself a function of the local curvature naturally leads to additional terms, such as those in the Kardar-Parisi-Zhang (KPZ) description. 30

Although it is clear that in the presence of a negative surface tension additional terms would be needed to stabilized the KPZ equation, we have tried to discriminate between different universality classes for the MIPS interface by measuring the critical exponents characterizing interfacial growth and steady state fluctuations. For $1 d$ interfaces both the EW and KPZ models are characterized by the same roughening exponent, $\alpha=1 / 2$, characterizing the growth of the steady-state interface width with system size $L_{y}$. They differ, however, in the exponent $\beta$ that controls the interface growth at short times, $w(t) \propto t^{\beta}$, with $\beta_{\mathrm{KPZ}}=1 / 3$ and $\beta_{\text {EW }}=1 / 4$. Discriminating between the two scenarios is numerically challenging for two reasons. First, the values of $\beta$ differ only slightly in the two models. Second, as previously pointed out, there is a smallest system size necessary to observe MIPS, so achieving even a single decade in linear system size $L_{y}$ requires very large computational effort. Nevertheless, we have run many short simulations, starting from a flat interface, to try to characterize the growth process. Surprisingly, we find that the EW dynamical exponent seem to better collapse the data than the (from a symmetry standpoint) more natural KPZ model. In particular, fits to $w(t)=A t^{\beta}$ for our largest systems suggest $\beta=0.23(3)$.

The key features of the MIPS interface seem to be a negative value of surface tension coupled with a scale-free, nearly equilibrium-like spectrum of interfacial fluctuations. The negative value of $\gamma$ suggests that either additional terms in $\partial_{t} h$ or the coupling of $h$ to another field is required. Natural candidates, such as $\nabla^{4} h$ terms or the coupling of the interfacial dynamics to a scalar field describing the flux of particles in and out of the two phases, select either a length scale or a time scale, and neither is seen in our system (we have confirmed that in our data the power spectrum of the interface $S(q, \omega)$ does not have any apparent time scale). The slight deviations from the equilibrium scaling of $\left\langle|\delta h(q)|^{2}\right\rangle$ may indicate that either a fundamentally nonlinear phenomenological model is required, or that simulations of much larger systems would reveal a long characteristic length scale in the problem. We view such simulations as a natural object of future study to resolve this issue.

\section{Appendices}

\section{A Locating the interface}

We have utilized two techniques to locate the interface and quantify its properties. The first uses a global frame of reference with axes normal and tangential to the mean (temporally and spatially averaged) interface ( $x$ and $y$ ) and yields a height map projected onto the $y$-axis, $h(y)$. The second traces an outline of the boundary of the strip yielding a parameterized curve $h(s)$ that captures fluctuations and overhangs. In both cases, for a given snapshot we quantify the configuration and position of left and right interface, denoted by $h_{\mathrm{L}, \mathrm{R}}$.
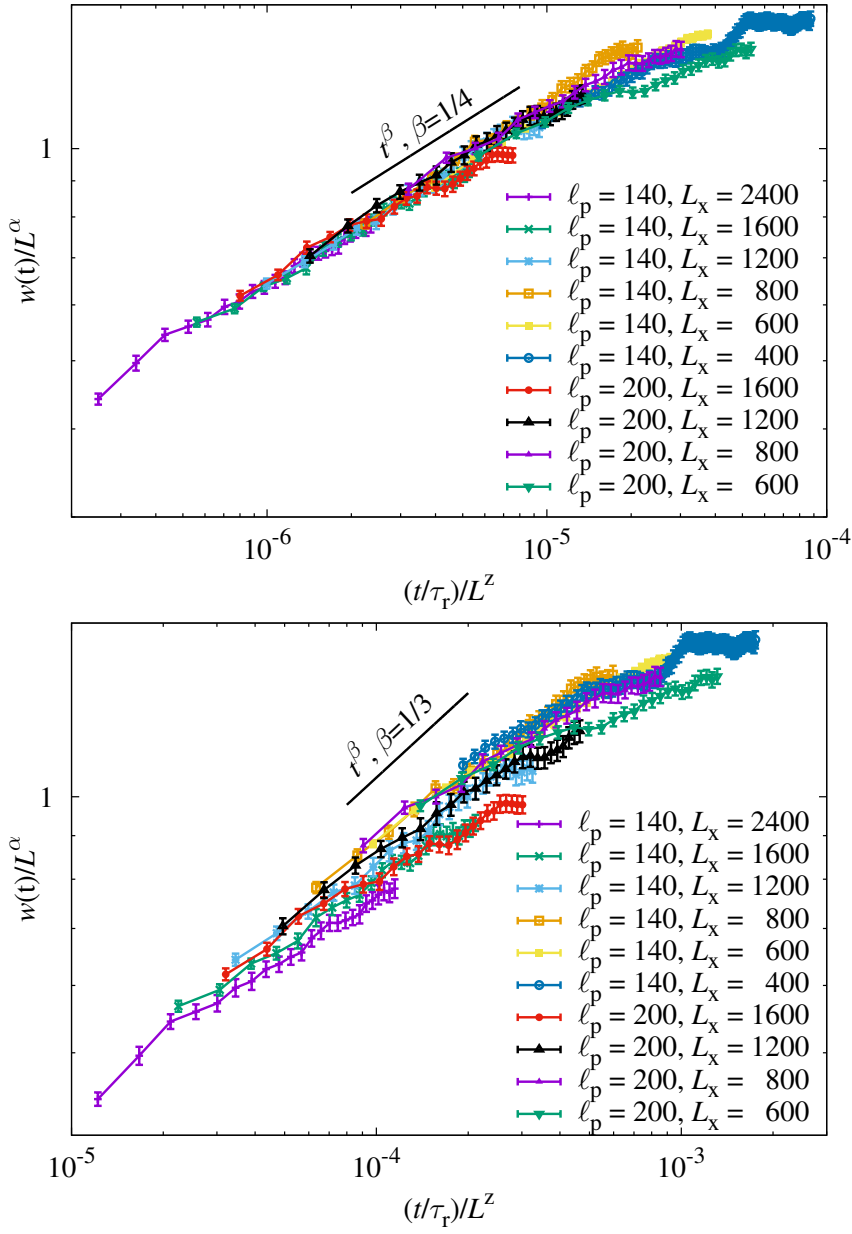

Fig. 6 The growth of the interfacial width $w(t)$ starting from a flat configuration for several $\ell_{\mathrm{p}}$ and system sizes is shown. We present a scaling collapse according to the Edwards-Wilkinson (top) and Kardar-ParisiZhang (bottom) critical exponents. Here $\alpha=1 / 2, z=\alpha / \beta, \beta_{\mathrm{KPZ}}=1 / 3$, $\beta_{\mathrm{EW}}=1 / 4$.

\section{A.1 Height map $h(y)$}

To calculate the height map we follow a straightforward procedure:

1. Distribute the cluster particles in $n_{\text {bin }}$ bins according to their $y$-position.

2. Sort particles in each bin according to their $x$-distance from the cluster center.

3. Average this $x$-distance of the left-most or right-most. $n_{\mathrm{avg}}$ particles. The resulting quantity is identified with $h_{\mathrm{L} / \mathrm{R}}(y)$. In general we use $n_{\mathrm{avg}}=3$.

The width $d_{y}=L_{y} / n_{\text {bins }}$ of the bins is chosen as $d_{y}=2 r_{0}$. We have also verified that slightly larger bins do not significantly change our measurements.

\section{A.2 Interface contour $\tilde{h}(s)$}

We have also quantified the interface not as a height map but as a curve parameterized by its arc length. To do so, we have implemented a classic contour-finding algorithm ${ }^{42}$ with square 


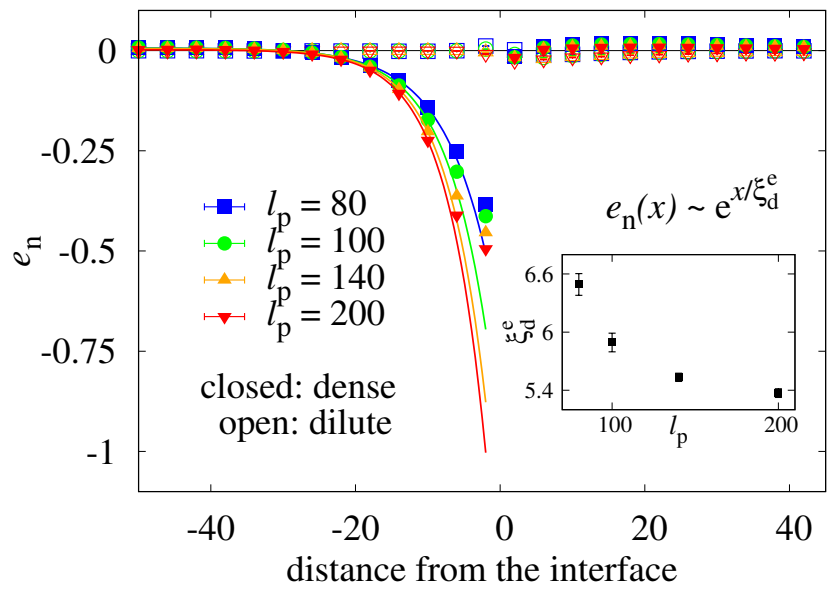

Fig. 7 The main figure shows the normal component of the polarization $e_{\mathrm{n}}$ measured in the local frame and averaged along the interface. This is proportional to the average local swim force per particle. The solid (open) symbols correspond to particles in the dense (gas) phase. The data show that there is an excess of inwardly polarized particles in a surface layer of thickness $\xi_{d}^{e}$ in the dense phase, but not in the gas. The length $\xi_{\mathrm{d}}^{\mathrm{e}}$ has been extracted with an exponential fit to the decay of $e_{\mathrm{n}}$ and is shown in the inset as a function of $\ell_{p}$. It is found to decay with increasing persistence. The net tangential component of polarization (not shown) remains zero at the interface because particles travel without preference in either direction tangent to the interface.

pixel resolution $l$ at the size of particles, $l=2 r_{0}$. Schematically, the algorithm proceeds as follows:

1. Discretize the system into square pixels of size $l$.

2. Mark all pixels containing cluster particles, call this set $G$.

3. Dilate $G$ by creating a new set $D$ such that $G \subset D$ where all pixels adjacent to members of $G$ are marked in $D$.

4. Determine set of contour pixels $C=D-G$.

5. Connect contours using a depth-first-search algorithm, collating adjacent points into subsets $c$ such that $c \in C$.

The result can be seen in Fig. 1. Once the sets $c$ are available, we locate the longest contours that cross the periodic boundaries an odd number of times. These are then sorted in left and right according to their $x$-distance from the strip center of mass $x_{\mathrm{CM}}$. The height $\tilde{h}(s)$ is then defined as the distance of these points from $x_{\mathrm{CM}}$.

\section{A.3 Smoothing}

While the above measure of the interface is generally useful for determining the interface length, including the more fractal inlets that exist down to the single-particle level, the noise on our measurements of local curvature is significantly reduced when we smooth the interface contours $\tilde{h}(s)$ to $h(s)$. To do this, we calculate the shortest path along $h(s)$ using the Dijkstra algorithm ${ }^{43}$ on this relatively sparse, but connected set. More specifically we

1. Choose any pixel $p \in c$ with only two neighbor pixels $p_{-}, p_{+} \in c$.

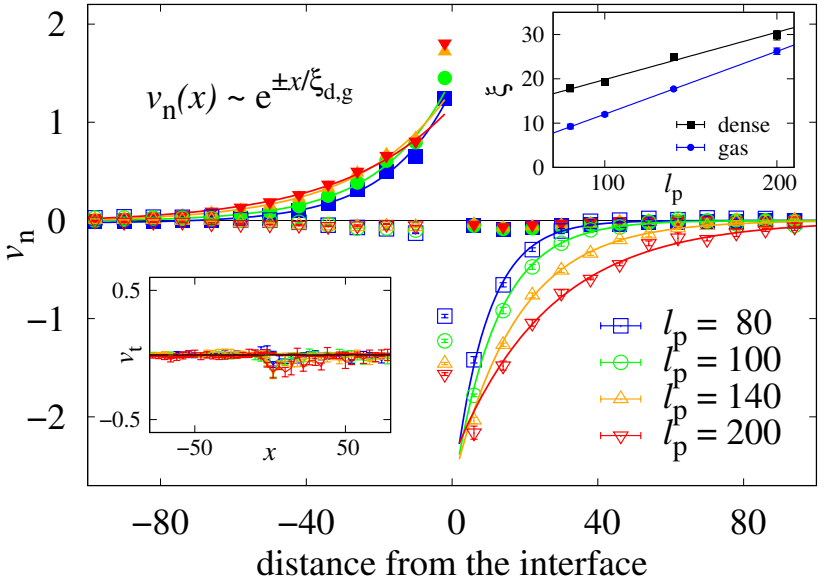

Fig. 8 The main figure shows the normal velocity $v_{\mathrm{n}}$ of particles measured in the local frame and averaged along the interface. The solid (open) symbols correspond to particles in the dense (gas) phase. The positive, outward-moving average velocity just inside the dense phase is balanced by the negative inward-moving average velocity just inside the gas phase. Within each phase, the normal average velocity is finite within a surface layer of thickness $\xi_{\mathrm{d}}$ (dense) and $\xi_{\mathrm{g}}$ (gas) and decays exponentially. The solid lines are fits to the exponential decay. The top right inset shows $\xi_{\mathrm{d}, \mathrm{g}}$ extracted from those fits as functions of $\ell_{p}$. The solid lines are fits that show the linear growth of $\xi_{\mathrm{d}, \mathrm{g}}$ with persistence. The bottom left inset shows that the signed tangential component of velocity is zero at the interface because particles travel without preference in either direction tangent to the interface.

2. Run Dijkstra on reduced contour $(c-p)$, starting at $p_{+}$and ending at $p_{-}$.

3. Add $p$ back to the Dijkstra path to connect the set $h(s)$.

The resulting $h(s)$ is now a smooth connected path that winds the periodic box in the $y$-direction.

\section{A.4 Determination of local normal to the interface}

Using either the basic contour $\tilde{h}(s)$ or the smoothed $h(s)$, we calculate a local normal $\hat{\mathbf{n}}$ using the local tangent vectors, as defined by each pixel and two nearest neighbors. We coarse-grain these vectors by averaging the normal vectors of the $n_{\text {cg }}$ nearest neighbors contour pixels. We test the result by eye and find that a coarse-graining of $n \approx 10$ neighboring pixels works well at several $\ell_{\mathrm{p}}$. The result is a set of normal vectors $\mathbf{n}(s)$ along the interface. For consistency, we use the same number of normals for the unsmoothed and smoothed interfaces, although this number could be reduced in the smoothed case.

\section{B Local frame}

Here we provide additional details of how we defined the local frame of the MIPS interface.

\section{B.1 Definition of frame}

The local frame is defined using $h(s)$ or $\tilde{h}(s)$ and the set of normal vectors $\mathbf{n}(s)$, where $s$ is the set of connected points defining the contour. Contours $\tilde{h}(s)$ are multiple-valued functions, so when using this contour we ignore those points that don't have enough 

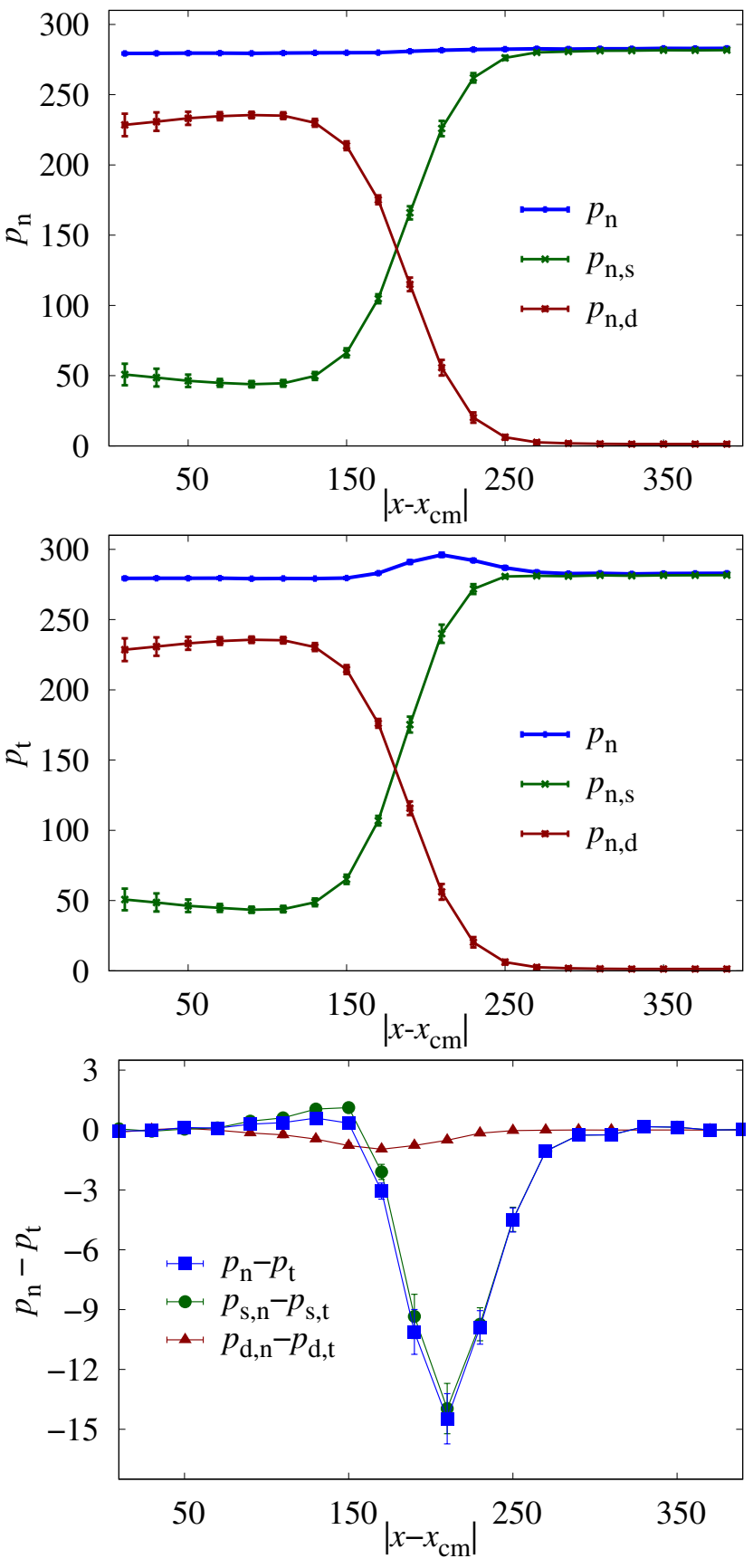

Fig. 9 Normal (top) and tangential (middle) pressure broken down into swim, interaction and total parts. The bottom panel shows the various contributions (total, swim and interaction) to the difference $p_{\mathrm{n}}-p_{\mathrm{t}}$ as a function of the distance $x$ from the dense phase center of mass, for $\ell_{p}=$ 140. This difference is the integrand in the expression for the tension, Eq. (11).

neighbors to properly define a normal. In the case of $\tilde{h}(s)$, this problem is avoided by definition.

Using $h(s)$ and $\mathbf{n}(s)$, we define a set of bins contained in a box of width $w_{\mathrm{c}}$ length $L_{\mathrm{c}}$, oriented along $\mathbf{n}(s)$ and centered at $h(s)$. The bin width $d n_{\mathrm{c}}=4 r_{0}$ sets the spatial resolution of our local frame data. A schematic of this frame is shown in Fig. 1 With this binning technique, we collect information for all particles that fall inside the box. Given the local normal, it is straightforward to properly transform the various scalar, vector and tensor physical quantities measured in this work from the global $(x-y)$ frame to the local frame.

\section{Local dynamics near the interface}

Previous work has focused on measuring the direction of the particles' propulsive force (polarization), identifying the excess of particles with inward polarization just inside the dense cluster as the main stabilizing mechanism. In this paper, we have examined in more detail the local dynamics in the interfacial region both within the dense and the gas phases. While we do observe an excess of particles with inward pointing polarization at the surface of the dense phase (see Fig. 7), the most intriguing observation is of sustained local tangential motion of particles in both regions. This dynamics is displayed in more detail in Fig 8 , where we show that the anisotropy of motion extends into each phase within a surface layer. Additionally, while the thickness of the region of inward-pointing polarization in the dense phase decreases with increasing persistence (see inset of Fig. 7), the thickness of the layers with finite particles velocity increases with persistence in both the gas and the dense phases. In these regions we observe both normal currents that balance each other and tangential currents that average to zero (Fig. 8), but greatly exceed in magnitude the normal currents (Fig. 4). This suggests that the stiffening of the interface results from the combined effect of inward polarization excess at the cluster's surface, local tangential flows in the gas, and the self-shearing of the interface in the dense cluster that heals fluctuations and enhances stability. This observation may provide additional intuition in constructing simple models of MIPS systems, such as the Active Matter Model $\mathrm{B}+28$.

\section{Conflicts of interest}

There are no conflicts to declare.

\section{Acknowledgments}

We thank Suraj Shankar and Michael Cates for helpful discussions. This work was primarily supported by NSF-DMR-1609208. Additional support was provided by NSF-POLS-1607416 (DMS), NSF-PHY-1748958 (DY, MCM) and FIS2015-65078-C2-1-P (DY), jointly funded by MINECO (Spain) and FEDER (EU). MCM and AP acknowledge support by the NSF IGERT program through award NSF-DGE-1068780. All authors acknowledge support of the Syracuse University Soft and Living Matter Program. Our simulations were carried out on a Tesla K40 donated by the NVIDIA corporation, on the Cierzo supercomputer (BIFI-ZCAM, Universidad de Zaragoza) and on the Syracuse University HTC Campus Grid, which is supported by NSF-ACI-1341006. MCM and DY thank the KITP for hospitality during part of this project.

\section{Notes and references}

1 P. G. de Gennes, Soft interfaces: the 1994 Dirac memorial lecture, Cambridge University Press, 2005.

2 Y. Fily and M. C. Marchetti, Physical Review Letters, 2012, 108, $1-5$.

3 J. Tailleur and M. E. Cates, Phys. Rev. Lett., 2008, 100, 218103. 
4 G. S. Redner, A. Baskaran and M. F. Hagan, Physical Review E, 2013, 88, 012305.

5 M. E. Cates and J. Tailleur, Annual Review of Condensed Matter Physics, 2015, 6, 150112144536003.

6 R. Wittkowski, A. Tiribocchi, J. Stenhammar, R. J. Allen, D. Marenduzzo and M. E. Cates, Nature communications, 2014, 5, 4351.

7 Y. Fily, S. Henkes and M. C. Marchetti, Soft matter, 2014, 10, 2132-40.

8 A. P. Solon, J. Stenhammar, R. Wittkowski, M. Kardar, Y. Kafri, M. E. Cates and J. Tailleur, Physical Review Letters, 2015, 198301, 1-6.

9 A. P. Solon, Y. Fily, A. Baskaran, M. E. Cates, Y. Kafri, M. Kardar and J. Tailleur, Nature Physics, 2015, 11, 673-678.

10 R. G. Winkler, A. Wysocki and G. Gompper, Soft Matter, 2015, 11, 6680-6691.

11 S. C. Takatori and J. F. Brady, Soft Matter, 2015.

12 U. Marini Bettolo Marconi, C. Maggi and S. Melchionna, Soft Matter, 2016, 12, 5727-5738.

13 A. P. Solon, J. Stenhammar, M. E. Cates, Y. Kafri and J. Tailleur, arXiv preprint arXiv:1803.06159, 2018.

14 G. S. Redner, M. F. Hagan and A. Baskaran, Physical Review Letters, 2013, 110, 1-5.

15 J. Bialké, H. Löwen and T. Speck, EPL (Europhysics Letters), 2013, 103, 30008.

16 X. Yang, M. L. Manning and M. C. Marchetti, Soft matter, 2014, 672, 6477-6484.

17 S. C. Takatori and J. F. Brady, Phys. Rev. E, 2015, 91, 032117.

18 I. Theurkauff, C. Cottin-Bizonne, J. Palacci, C. Ybert and L. Bocquet, Phys. Rev. Lett., 2012, 108, 268303.

19 I. Buttinoni, J. Bialké, F. Kümmel, H. Löwen, C. Bechinger and T. Speck, Phys. Rev. Lett., 2013, 110, 238301.

20 J. Palacci, S. Sacanna, A. P. Steinberg, D. J. Pine and P. M. Chaikin, Science, 2013, 339, 936-940.

21 S. A. Mallory, A. Šarić, C. Valeriani and A. Cacciuto, Phys. Rev. E, 2014, 89, 052303.

22 C. Bechinger, R. Di Leonardo, H. Löwen, C. Reichhardt, G. Volpe and G. Volpe, Rev. Mod. Phys., 2016, 88, 045006.

23 G. Liu, A. Patch, F. Bahar, D. Yllanes, R. D. Welch,
M. C. Marchetti, S. Thutupalli and J. W. Shaevitz, arXiv:1709.06012, 2017.

24 J. Bialké, J. T. Siebert, H. Löwen and T. Speck, Physical Review E, 2015, 098301, 1-5.

25 T. Speck, EPL (Europhysics Letters), 2016, 114, 30006.

26 C. F. Lee, Soft matter, 2017, 13, 376-385.

27 S. Paliwal, V. Prymidis, L. Filion and M. Dijkstra, The Journal of Chemical Physics, 2017, 147, 84902.

28 E. Tjhung, C. Nardini and M. E. Cates, arXiv preprint arXiv:1801.07687, 2018.

29 S. F. Edwards, D. Wilkinson et al., Proc. R. Soc. Lond. A, 1982, pp. 17-31.

30 M. Kardar, G. Parisi and Y.-C. Zhang, Physical Review Letters, 1986, 56, 889.

31 M. C. Marchetti, Y. Fily, S. Henkes, A. Patch and D. Yllanes, Current Opinion in Colloid \& Interface Science, 2016, 21, 3443.

32 J. S. Rowlinson and B. Widom, Molecular theory of capillarity, Courier Corporation, 2013.

33 J. G. Kirkwood and F. P. Buff, The Journal of Chemical Physics, 1949, 17, 338-343.

34 A. Patch, D. Yllanes and M. C. Marchetti, Physical Review E, 2017, 95, 012601.

35 Y. Fily, Y. Kafri, A. P. Solon, J. Tailleur and A. Turner, Journal of Physics A: Mathematical and Theoretical, 2017, 51, 044003.

36 S. C. Takatori and J. F. Brady, Soft matter, 2014, 9433-9445.

37 J. A. Anderson, C. D. Lorenz and A. Travesset, J. Comput. Phys., 2008, 227, 5342.

38 HOOMD-blue, https://codeblue.umich.edu/ hoomd-blue/ 2017.

39 C. del Junco and S. Vaikuntanathan, arXiv:1803.02678, 2018.

40 Y. Fily, A. Baskaran and M. F. Hagan, Soft Matter, 2014, 10, 5609-5617.

41 Y. Fily, A. Baskaran and M. F. Hagan, The European Physical Journal E, 2017, 40, 61.

42 G. Toussaint, Grids, Connectivity and Contour Tracing, accessed: 1 November, 2017.

43 T. Cormen, C. Leiserson, R. Rivest and C. Stein, Introduction to Algorithms, MIT Press, 3rd edn, 2009. 